\title{
PSICOLOGIA COGNITIVO-COMPORTAMENTAL E EXPERIÊNCIA DE ESTÁGIO EM PSICOLOGIA CLÍNICA: AVALIAÇÃO PSICOLÓGICA DA ANSIEDADE
}

\author{
Júlio Gonçalves ${ }^{1 *}$; Andressa Juliana de Oliveira ${ }^{1}$; \\ Juliana Vieira A. Silva ${ }^{2}$
}

\begin{abstract}
RESUMO
GONÇALVES, J.; OLIVEIRA, A.J.; SILVA, J.V.A. Psicologia Cognitivo-Comportamental e experiência de estágio em Psicologia Clínica: avaliação psicológica da Ansiedade. Perspectivas Online: Humanas \& Sociais Aplicadas, v. 8, n.21, p.28-36,2018.
\end{abstract}

O presente artigo consiste no relato de experiência ocorrido na Clínica Escola do Curso de Psicologia de uma Universidade Comunitária, e tem por objetivo, descrever o processo de avaliação psicológica realizado com paciente $(\mathrm{L})$ que obteve psicodiagnóstico de Fobia Específica Situacional com comorbidade ao Transtorno de Ansiedade Social do tipo somente desempenho. A aquisição dos dados se deu por meio da entrevista de triagem inicial e aplicação de testes psicológicos: o Inventário de Ansiedade do Beck (BAI), o
Inventário de Habilidades Sociais (IHS) e o Inventário de Estilos Parentais (IEP), ambos selecionados com base nas informações da entrevista inicial. A partir dos resultados advindos dos testes psicológicos e da observação clínica, foi realizada Conceitualização Cognitiva e interpretação dos dados a partir da perspectiva teórica Cognitivo-Comportamental, e posteriormente realizado a devolutiva $\mathrm{e}$ encaminhamento do paciente.

Palavras-chave: Distúrbios da Ansiedade; Fobias; Avaliação Psicológica.

ABSTRACT
$\begin{aligned} & \text { The purpose of this article is to describe the (BAI), the Social Skills Inventory (IHS) and the } \\ & \text { psychological evaluation process performed with a }\end{aligned}$ Parenting Styles Inventory (IEP), both selected
patient (L) who obtained psychodiagnosis of with based on initial interview information. From
Situational Specific Phobia with comorbidity to the the results of the psychological tests and the
Disorder of Social anxiety only performance type. clinical observation, the data interpretation was
The data acquisition was done through the performed from the cognitive-behavioral
interview of initial screening and application of theoretical perspective, and later performed the
psychological tests: the Beck Anxiety Inventory devolution and referral of the patient.

Keywords: Anxiety Disorders; Phobias; Psychological evaluation.

\footnotetext{
${ }^{1}$ Acadêmicos da Universidade do Vale do Itajaí - UNIVALI, Escola de Ciências da Saúde, Curso de Psicologia, Rua Uruguai, 458, Itajaí, SC, CEP: 88302-901, Brazil.

${ }^{2}$ Docente da Universidade do Vale do Itajaí - UNIVALI, Escola de Ciências da Saúde, Curso de Psicologia, Rua Uruguai, 458, Itajaí, SC, CEP: 88302-901, Brazil

(*)e-mail: psico.julio@edu.univali.br

Data de chegada: 16/04/2018 Aceito para publicação: 12/05/2018
} 


\section{INTRODUÇÃO}

A Psicologia é uma formação relativamente recente no Brasil, tendo sido regularizada como profissão somente em meados dos anos 60 , e, concomitante a sua regularização, emergiram as Clínicas-Escolas as quais têm como principal objetivo, atender à necessidade da formação prática em faculdades e universidades nas quais esteja presente o curso de Psicologia (AMARAL, 2012).

A Clínica-Escola em Psicologia consiste no ambiente vinculado a uma instituição de ensino, em que o acadêmico, para concluir sua formação, tem como exigência curricular o Estágio Obrigatório na Prática Clínica sob a orientação de um professor supervisor. Este estágio possui por objetivos a promoção de ações, experiências e procedimentos que possibilitem o ensino e a pesquisa no nível prático, colaborando para a formação do aluno ao mesmo tempo que possibilita o acesso gratuito à população de baixa renda econômica aos atendimentos psicológicos (MARAVIESKI; SERRALTA, 2011). Os Estágios Obrigatórios visam assegurar a consolidação e a articulação das competências estabelecidas pela Resolução $\mathrm{CNE} / \mathrm{CES} \mathrm{n}^{\circ} 5$, de 15 de março de 2011, que institui as Diretrizes Curriculares Nacionais para os cursos de graduação em Psicologia, estabelecendo normas para o projeto pedagógico complementar para a Formação de Professores de Psicologia (BRASIL, 2011).

Por conseguinte, dentre as múltiplas aprendizagens e experiências possibilitadas pelas Clínicas-Escolas durante o processo psicoterapêutico, encontra-se a Avaliação Psicológica. Esta se constitui em um procedimento clínico endossado por um organizado princípio teórico, metodológico e técnico de investigação. O processo avaliativo é o princípio norteador para a escolha do tratamento mais adequado no processo terapêutico, principalmente quando voltado para a avaliação de um construto como a ansiedade (PRIMI, 2010).

Os indivíduos que iniciam atendimento psicológico procuram espaços para serem acolhidos, não tendo conhecimento em grande parte das vezes acerca do tratamento que necessitam, e tal busca caracteriza-se como um pedido de ajuda, o que torna primordial o acompanhamento efetivo do psicólogo no decorrer do processo psicoterápico. $\mathrm{O}$ acolhimento tem significado importante para o paciente, visto que a postura afetiva e empática do psicólogo proporciona alívio, transformando o inominável em palavras, permitindo assim a elaboração de pensamentos com conteúdos de que há soluções para seus problemas (CERIONI; HERZBERG, 2016).

No início do processo de atendimento psicoterapêutico, faz-se necessária a realização de uma triagem, sendo esta considerada mais do que uma simples coleta de dados, pois além de ser organizada a partir de um roteiro, promove uma intervenção breve que o terapeuta engaja e responsabiliza o paciente a conhecer os próprios problemas e motivos que o fizeram procurar auxílio; bem como proporciona ao terapeuta a possibilidade de desenvolver uma aliança terapêutica segura, conforme os princípios das terapias cognitivas (BECK, 2013).

Alusivo especificamente à avaliação psicológica nos transtornos de ansiedade, a coleta pode ser realizada por meio de questionários, escalas, inventários psicométricos, análise cognitivo-comportamental e observação direta. O processo de avaliação psicológica deve se embasar em instrumentos subsidiados teoricamente e respaldados por evidências empíricas, de modo a possibilitar a elaboração de diagnósticos e prognósticos seguros, ambos necessários para a continuidade efetiva da psicoterapia (DE SOUZA et al., 2013).

No quesito de modelos teórico-práticos, a Terapia Cognitivo-Comportamental (TCC) tem apresentado evidências substanciais no tratamento dos transtornos ansiosos, e detêm, segundo os estudos psicossociais baseados em evidências, efetividade semelhante à terapia farmacológica para Transtorno de Ansiedade Generalizada e provável eficácia e eficácia bem estabelecida no uso das técnicas de relaxamento, resolução de problemas, controle antecipatório de preocupação, catastrofização e foco no tratamento para a mudança (HOFMANN; SMITS, 2008; DEWES et al., 2010; HOFMANN et al., 2012). Por conseguinte, para

Persp. online: hum. \& sociais aplicada., Campos dos Goytacazes, 21 (8), 28-36, 2018

seer.perspectivasonline.com.br 
selecionar os métodos interventivos a partir do aporte teórico é necessário que a avaliação psicológica contemple de maneira fiel as queixas, os déficits e os problemas do paciente.

No que concerne o modelo teórico cogntivo-comportamental, sua premissa básica se fixa na compreensão de que as percepções que o indivíduo detém sobre o mundo, alteram comportamentos, sentimentos e emoções. Essas percepções podem ser identificadas através dos pensamentos automáticos que se caracterizam por interpretações imediatas de qualquer situação. Os pensamentos automáticos são mantidos por esquemas cognitivos, compostos por crenças nucleares que se alicerçam através das experiências adquiridas durante o processo de desenvolvimento, e geram, consequentemente, crenças subjacentes com pressupostos baseados em regras, atitudes e suposições que mantém sua interpretação acerca dos contextos que acessa (KNAPP; BECK, 2008; BECK, 2013).

Desta forma, concomitante a avaliação psicológica por meio de testes, a TCC se utiliza da Conceitualização Cognitiva, que se caracteriza em um diagrama na qual são dispostos os esquemas de crenças dos indivíduos, a partir de situações do cotidiano que possuem valor aversivo e geradores de sofrimento, para assim traçar o plano de tratamento (HOFMANN, 2014).

Face ao exposto, este artigo tem como objetivo discorrer sobre a experiência de estágio na ClínicaEscola do Curso de Psicologia de uma Universidade Comunitária. Como objetivos específicos, será descrito o processo de avaliação psicológica realizado com um paciente que obteve como psicodiagnóstico, embasado no Manual Diagnóstico e Estatístico de Transtornos Mentais - DSM-5 (APA, 2014), a Fobia Específica Situacional com comorbidade ao Transtorno de Ansiedade Social do tipo somente desempenho. Especificamente, discorreu-se sobre os resultados dos testes psicológicos utilizados, a avaliação cognitivocomportamental a partir do modelo explicativo Cognitivo-Comportamental, e a devolutiva com os devidos encaminhamentos para posteriormente ser realizada a intervenção psicológica.

\section{METODOLOGIA}

\subsection{Local, participantes e procedimentos éticos}

O presente trabalho consiste em um estudo descritivo, do tipo relato de experiência. Esta tipo de metodologia tem por objetivo descrever características e propriedades de um fenômeno (SAMPIERI; COLLADO; LUCIO, 2014). Os atendimentos ocorrem nas dependências da Clínica-Escola do Curso de Psicologia de uma Universidade Comunitária, desenvolvido entre meses de setembro a dezembro de 2016. O paciente buscou atendimento na Clínica-Escola de forma espontânea e será abordado no artigo sob o acrônimo (L.). Suas queixas referiam-se às frequentes respostas de ansiedade e medo na realização de viagens de ônibus intermunicipais de longa distância, e um padrão constante de antecipação do futuro diante dos contextos em que exigem seu desempenho.

A avaliação psicológica iniciou-se após autorização por meio do Termo de Consentimento Livre e Esclarecido (TCLE) que consta a utilização das sessões para fins de pesquisas acadêmicas. O TCLE (documento suplementar) possui um modelo padrão fornecido pela Clínica Escola de Psicologia durante as práticas de Estágios Obrigatórios, em que este, em uma de suas cláusulas, cita que os registros durante os procedimentos psicoterápicos podem ser utilizadas, tanto por acadêmicos Estagiários do curso de Psicologia, quanto por Professores Orientadores do referido Curso, por Psicólogos legalmente habilitados vinculados a pesquisas devidamente aprovadas pelo Comitê de Ética em Pesquisa da Universidade, como também pela Equipe Técnica da Clínica de Psicologia da Universidade, em pesquisas científicas vinculadas ao Comitê de Ética em Pesquisa, bem como em publicações em Revistas Científicas e Periódicos especializados e apresentações em eventos científicos.

\subsection{Instrumento para coleta de dados}

Para aquisição de dados realizou-se a entrevista de triagem, entrevista inicial e aplicação de testes psicológicos. Posterior à entrevista clínica, os dados organizaram-se de acordo com a anamnese, instrumento que auxilia o terapeuta a organizar detalhadamente o processo de desenvolvimento do sujeito de forma cronológica, tendo por finalidade auxiliar o levantamento da hipótese diagnóstica (Cunha, 2000). De modo a

Persp. online: hum. \& sociais aplicada., Campos dos Goytacazes, 21 (8), 28-36, 2018

seer.perspectivasonline.com.br 
complementar a avaliação inicial coletada por meio da entrevista, aplicou-se o Inventário de Ansiedade do Beck (BAI), Inventário de Habilidades Sociais (IHS) e Inventário de Estilos Parentais (IEP) tendo sua escolha feita a partir de dados na entrevista inicial.

O BAI é uma escala de auto relato com 21 itens, tendo 4 alternativas e com escores de 0 a 3 , caracterizando os graus de intensidade dos sintomas ansiosos. Os tópicos avaliam sintomas fisiológicos como dormência, taquicardia, tremores, frequência cardíaca, e comportamentais. Na versão em português os pontos de corte se dividem em: 0 a $10=$ mínimo, de 11 a $19=$ leve, de 20 a $30=$ moderado e de 31 a $63=$ grave (CUNHA, 2001).

O IHS engloba uma série de contextos, diversos locutores e variadas demandas sociais para avaliação das habilidades sociais. $\mathrm{O}$ instrumento contém 38 itens, cada um contendo afirmações referentes a possíveis respostas comportamentais, fisiológicas e emocionais. No item de frequência as respostas variam entre nunca ou raramente, com pouca frequência, com regular frequência, muito frequentemente e sempre ou quase sempre (DEL PRETTE; DEL PRETTE, 2001).

O IEP permite a visualização das atitudes administradas pelos pais advindos de um modelo teórico composto por sete práticas educativas, sendo duas consideradas positivas, monitoria positiva e comportamento moral, e cinco negativas, abuso físico, disciplina relaxada, monitoria negativa, negligência e punição inconsistente. As práticas são avaliadas por intermédio de 6 questões cada, em um total de 42 afirmativas. As respostas são dadas em uma escala de likert de 3 pontos. O IEP fornece um escore da subtração da soma das práticas negativas da soma das positivas. Os percentis são agrupados nas categorias de estilo parental ótimo, regular acima da média, regular abaixo da média, e de risco (GOMIDE, 2006).

Como estratégia para o acolhimento de dados, parte das sessões eram organizadas em formas de questionamentos, formuladas com base nos diálogos e análises obtidas até então, com posterior aplicação de testes psicológicos.

\section{RESULTADOS}

$\mathrm{Na}$ aplicação do IHS o adolescente obteve um percentil, no escore total, de $20 \%$ o que segundo Del Prette e Del Prette (2001), se configura abaixo da mediana sendo indicado o treinamento em Habilidades Sociais (HS) quando os déficits se tornam fonte dos problemas. Nos escores fatoriais, três conjuntos de habilidades obtiveram um percentil abaixo da média: autoafirmação na expressão de sentimento positivo (F2), conversação e desenvoltura social (F3), e auto exposição a desconhecidos e situações novas (F4). O escore do fator de enfrentamento e autoafirmação com risco (F1) obteve um percentil mediano, e o fator de autocontrole da agressividade (F5) acima da média.

$\mathrm{Na}$ aplicação do BAI, o adolescente obteve um escore de 22 pontos, caracterizando um nível de ansiedade moderado (CUNHA, 2001). Parte das afirmações obteve a resposta moderadamente nas emissões de respostas fisiológicas, tendo se destacado de ordem grave o medo de perder o controle. Quando discutido cada item do BAI, evidenciou-se que os sintomas de ansiedade se intensificam consideravelmente quando é necessário a apresentação de seu desempenho em alguma tarefa.

$\mathrm{Na}$ aplicação do IEP, evidenciou-se um percentual de $35 \%$ na figura materna, considerado um estilo parental regular, porém abaixo da média. A figura paterna obteve-se um percentual de $65 \%$, considerado um percentual bom, acima da média (GOMIDE, 2006). O fator punição inconsistente (C), disciplina relaxada (E) e monitoria negativa $(\mathrm{F})$ referente a figura materna, se destacaram como índices mais baixos e deficitários, em que de modo geral, retratam as regras e limites que são inseridos ao longo do processo de investimento parental. Os outros fatores não se destacaram de maneira positiva a ponto de neutralizar esses fatores deficitários, sendo indicado a participação de grupos terapêuticos para treinamento de práticas parentais (GOMIDE, 2006).

Apesar da figura paterna ter se destacado de maneira negativa nos fatores comportamento moral (B), punição inconsistente (C), negligência (D) e abuso físico (G), estas, além de terem atingido um percentual perto da média, são neutralizadas pelo investimento nos outros fatores, permanecendo de modo geral acima

Persp. online: hum. \& sociais aplicada., Campos dos Goytacazes, 21 (8), 28-36, 2018

seer.perspectivasonline.com.br 
da média, sendo indicado a leitura de livros de orientação para pais visando aprimoramento da prática parental (GOMIDE, 2006).

Concomitante à aplicação dos testes psicológicos e relatos fornecidos pelo paciente, foi elaborado a Conceitualização Cognitiva (HOFMANN, 2014) a partir do modelo Cognitivo Comportamental, conforme pode ser observado na figura 1 :

\begin{tabular}{|c|c|c|}
\hline \multicolumn{3}{|c|}{$\begin{array}{l}\text { DADOS RELEVANTES } \\
\text { ntomas ansiosos principiaran }\end{array}$} \\
\hline \multicolumn{3}{|c|}{$\begin{array}{c}\text { CRENÇAS CENTRAIS } \\
\text { Si: Não sou bom para conversar; preciso fazer tudo certo; não sou bom em nada } \\
\text { Mundo: Se eu não for bom em tudo as pessoas irão me rejeitar } \\
\text { Futuro: Meu futuro será bom se eu fizer tudo certo e o melhor possivel }\end{array}$} \\
\hline \multicolumn{3}{|c|}{$\begin{array}{c}\text { PRESSUPOSTOS SUBJACENTES } \\
\text { Atitude: É ruim não conseguir ser bom em tudo } \\
\text { Regra: Preciso fazer tudo certo para ser aceito } \\
\text { Suposição: Se eu errar, então as pessoas vão brigar comigo }\end{array}$} \\
\hline \multicolumn{3}{|c|}{$\begin{array}{l}\text { ESTRATÉGIAS COMPENSATÓRIAS } \\
\text { Pensamentos automáticos frequentes com conteúdos de antecipação do futuro e } \\
\text { alto grau de esquiva em quaisquer situações em que acredita ser incapaz de } \\
\text { enfrentar, mantendo um padrão perfeccionista para todas atividades que executa }\end{array}$} \\
\hline $\begin{array}{c}\text { Situação } 1 \\
\text { Recebimento de } \\
\text { pagamento de um cliente } \\
\text { da empresa em que } \\
\text { trabalha em local indevido }\end{array}$ & \begin{tabular}{|} 
Situação 2 \\
Entrega de trabalho da \\
disciplina e os colegas não \\
executaram sua parte
\end{tabular} & $\begin{array}{c}\text { Situação 1 } \\
\text { Dores abdominais, êmese } \\
\text { e permanência na } \\
\text { emergência hospitalar } \\
\text { durante viagem de longa } \\
\text { distância }\end{array}$ \\
\hline $\begin{array}{l}\text { Pensamento Automático } \\
\text { Se eu não receber o } \\
\text { pagamento aqui vou } \\
\text { parecer incompetente }\end{array}$ & \begin{tabular}{|} 
Pensamento Automático \\
Vou fazer o trabalho para \\
evitar nota baixa e \\
discussão com meus \\
colegas
\end{tabular} & $\begin{array}{l}\text { Pensamento Automático } \\
\text { Viagens longas são } \\
\text { perigosas, ainda mais } \\
\text { sozinho }\end{array}$ \\
\hline $\begin{array}{l}\text { Significado do PA } \\
\text { Vulnerabilidade } \\
\text { Incapacidade }\end{array}$ & $\begin{array}{l}\text { Significado do PA } \\
\text { Baixa assertividade }\end{array}$ & $\begin{array}{l}\text { Significado do PA } \\
\text { Desamparo } \\
\text { Vulnerabilidade }\end{array}$ \\
\hline
\end{tabular}

Figura 1: Diagrama da Conceituação Cognitiva.

Fonte: Adaptado de ANDRETTA, I.; OLIVEIRA, M. S. Manual Prático de

Terapia Cognitivo-Comportamental. Editora Pearson, 2011.

\section{DISCUSSÃO}

A partir dos dados coletados e interpretação dos mesmos a partir do modelo CognitivoComportamental, as dificuldades e déficits de L. se caracterizam em uma série de esquivas na manifestação de suas opiniões, agindo com pouca assertividade na expressão de seus sentimentos e com baixa habilidade

Persp. online: hum. \& sociais aplicada., Campos dos Goytacazes, 21 (8), 28-36, 2018

seer.perspectivasonline.com.br 
em rejeitar pedidos abusivos. Corrborando com a literatura, uma das característica que define os transtornos ansiosos é a preocupação. Diferente da antencipação de um evento possivelmente aversivo, o ato de preocupar-se envolve atividade verbal e imagens, que são processos cognitivos. Tais processos cognitivos, por sua vez, inibem o processamento emocional do conteúdo aflitivo, portanto, preocupar-se pode ser considerado uma forma de esquiva cognitiva. Ao preocupar-se, o indivíduo evita pensar a respeito de um evento mais catastrófico (HOFMANN, 2014).

Alusivo à identificação entre a diferença de uma ansiedade aceitável e disfuncional, esta ocorre por meio da avaliação das estratégias de enfrentamento desenvolvida pelo sujeito e o quantos estas geram sofrimento psicológico ao indivíduo (HOFMANN, 2014). No caso de L. os comportamentos acima possuem função punitiva tanto na vida pessoal quanto profissional, pois o paciente possui como estratégias compensatórias frequentes esquivas e pensamentos automáticos com conteúdo de antecipação do futuro quando exposto a contextos que antecedem eventos aversivos. Seus pressupostos se fixam na necessidade de executar o melhor possível as solicitações de outras pessoas, quaisquer indícios de falha ocasionam pensamentos catastróficos seguidos de alta taxa de respostas ansiosas, em que a intolerância ao atraso de reforçadores se estendem à vida escolar, profissional e amorosa, ocasionando uma série de contratempos e afetam negativamente o dia-a-dia de $\mathrm{L}$.

Outra característica visível nos transtornos ansiosos é a emissão de múltiplos comportamentos que possuem a função de fuga e esquiva de eventos potencialmente aversivos, os quais, consequentemente, reforçam as crenças de periculosidade incompatíveis com o que realmente ocorre (ZORTEA, 2012). As crenças se mantém por abstrações seletivas, onde o indivíduo retém somente informações que confirmam a crença disfuncional, e catastrofização que se caracteriza por uma previsão negativa dos eventos futuros (BECK, 2013). De modo geral, grande parte dos contextos nos quais L. se insere são considerados aversivos, havendo frequente padrão de antecipação desses eventos, com taxas altas de ansiedade.

Referente à dinâmica familiar, a mãe do paciente se apresenta como uma figura superprotetora, mantendo-se constantemente vigilante aos passos de seu filho e atendendo a maioria de seus pedidos. Consequentemente, L., diante do argumento de que nunca precisou tomar decisões e que sempre está acompanhado da mãe nos lugares em que frequenta, possui dificuldades em sair sozinho na rua, antecipando seguidamente um futuro catastrófico mantidas por crenças rígidas de que será assaltado. Os repertórios de medo dos pais também são variáveis que podem se tornar um modelo para os filhos, o que desencadeia o desenvolvimento de esquivas e fugas constantes em diversas áreas da vida, na qual a crença de que tudo é perigoso no mundo torna-se um princípio norteador para atuar nos ambientes que acessa (ZORTEA, 2012).

Outro aspecto relevante da queixa é que L. costumeiramente anda de ônibus, carro, vans, em viagens de curta distância e não há problemas, porém, ao utilizar ônibus para realização de viagens de longa distância, principalmente intermunicipais, há uma ampla intensidade de comportamentos ansiosos, pensamentos catastróficos, desconforto abdominal e consequente êmese, ambas causando intenso sofrimento ao paciente. Mediante relato do paciente, pode-se evidenciar que a manutenção desse contexto, ocorre por meio de condicionamento respondente em que o estímulo inicialmente neutro "viagem de longa distância" e seus constituintes como a estadia prolongada em ambiente fechado, motorista, ônibus de dois andares, estímulos olfativos, entre outros, pareado as sensações de mal-estar, desconforto abdominal e êmese, estes chamados de estímulos aversivos incondicionados, permitem que contextos similares a "viagem de longa distância" adquirem propriedades aversivas em contextos diferentes, compreendido a partir do princípio de generalização de estímulos (DE FARIAS, et al., 2017). As respostas de medo ocorrem de maneira antecipada e frequente por meio da rememoração, mantendo um padrão intenso de pensamentos catastróficos, respostas fisiológicas e comportamentais de esquiva, se caracterizando em alta taxa de ansiedade, o que afeta marcadamente os momentos em que necessita realizar viagens.

Por conseguinte, a partir dos dados coletados via testes psicológicos, Conceitualização Cognitiva e discussão a partir do modelo Cognitivo-Comportamental, os sintomas expressos por L. se constituem com base no Manual Diagnóstico e Estatístico de Transtornos Mentais DSM-5 (APA, 2014), na hipótese diagnóstica de Fobia Específica Situacional com comorbidade ao Transtorno de Ansiedade Social do tipo somente desempenho.

Persp. online: hum. \& sociais aplicada., Campos dos Goytacazes, 21 (8), 28-36, 2018 
Segundo o DSM V, o medo ou ansiedade acentuados acerca de situações sociais se caracterizam em Transtorno de Ansiedade Social (TAS), podendo ser especificado do tipo somente desempenho (APA, 2014). O paciente atende todos critérios para diagnóstico, os quais são: a) medo ou ansiedade quando exposto a situações sociais e há possibilidade de avaliação de outras pessoas; b) o indivíduo teme agir de forma que demostre seus comportamentos ansiosos e possível avaliação negativa; c) as situações sociais causam medo ou ansiedade; d) As situações sociais são evitadas ou suportadas com intenso sofrimento; e) o medo ou ansiedade é desproporcional à ameaça apresentada; f) o medo, ansiedade ou esquiva é persistente durante mais de seis meses; g) o medo, ansiedade ou esquiva causam prejuízo no funcionamento social, profissional e outras áreas relevantes para o indivíduo; h) o medo, ansiedade ou esquiva não são consequências fisiológicas do uso de substâncias ou de outra condição médica; i) a situação não é melhor explicada por outro transtorno mental; j) se outra condição médica está presente, o medo, ansiedade ou esquiva não é relacionado.

A especificação do tipo desempenho se configura em preocupações do indivíduo com sua performance na vida profissional e situações que requer falar em público. Os medos e comportamentos ansiosos se manifestam nos contextos profissionais, escola, interpessoal, não evitando situações que não envolvam desempenho. $\mathrm{O}$ medo e ansiedade elevados quando exposto a estímulos específicos, com duração mínima de seis meses, se caracterizam em uma Fobia Específica (FE) segundo o DSM-5 (APA, 2014). Os critérios que L. preenche se constituem em: a) medo ou ansiedade acentuados acerca de um objeto ou situação; b) o estímulo fóbico gera respostas imediatas de medo ou ansiedade; c) O estímulo fóbico é evitado ou suportado com significativo sofrimento; d) $\mathrm{O}$ medo, ansiedade ou esquiva são persistentes, prevalecendo nos últimos seis meses; e) O medo, ansiedade ou esquiva causam prejuízo no funcionamento social, profissional e outras áreas relevantes para o indivíduo; f) A situação não é melhor explicada por outro transtorno mental.

Para tratamento da Fobia Específica e o Transtorno de Ansiedade Social sugeriu-se a continuidade da Psicoterapia Cognitivo-Comportamental. A Teoria Cognitivo-Comportamental (TCC) é uma abordagem que detém inúmeras pesquisas validando sua eficácia como forma de tratamento, se estendendo a diversos transtornos psicológicos (POWELL et al., 2008). Considerou-se também a Resolução 007/2003 do Conselho Federal de Psicologia (CFP, 2003) na citação de que os objetivos do processo de avaliação psicológica têm determinações históricas, sociais, econômicas e políticas, sendo as questões de ordem psicológicas constituídas neste processo de subjetivação. Sendo assim, é considera-se a natureza dinâmica, não decisiva e não cristalizada do objeto de estudo.

Vale ressaltar que o fator de risco de ordem ambiental que contribui para aquisição dos sintomas elevados de ansiedade, ao estar diante de estímulos específicos, é a prática parental de proteção exacerbada (APA, 2014). A adolescência por se caracterizar em um estágio em que a socialização, as regras sociais, as instruções formais, os elementos culturais passam a serem explorados e refinados, pode gerar desconforto aos pais, por não deterem controle sobre as contingências em que o filho acessa. É nesse período que o relacionamento com elementos do mundo se torna mais intenso na medida em há interação (Bronfenbrenner, 2002).

Para que o adolescente possa desenvolver um padrão de comportamentos mais funcionais, são requeridas habilidades sociais específicas para resolver os problemas diários, que se resume em estar diante de eventos estressantes e se adaptar a nível comportamental a essas novas demandas, tanto em casa, na universidade e profissionalmente. Essa mudança por si só desencadeia novos comportamentos e o que ditará se esses são adequados ou não é a função em que eles adquirem na vida das pessoas. No caso de L., a resposta de êmese na realização de viagens intermunicipais, a esquiva constante de locais considerados arriscados e a alta taxa de ansiedade na antecipação de eventos futuros têm afetado consideravelmente sua rotina, o que faz com que seus desempenhos nas atividades básicas de seu dia a dia estejam defasados.

\section{CONCLUSÕES}

Dentre as competências científico-profissionais que devem ser ensinadas e aprimoradas durante o percurso de formação do psicológo estão a identificação de necessidades por meio de processos de investigação e/ou avaliação e a comunicação do trabalho realizado com base no conhecimento obtido com objetivo de promover mudanças ou melhorias naquilo que foi demandado (CRUZ, 2016). Essa asserção

Persp. online: hum. \& sociais aplicada., Campos dos Goytacazes, 21 (8), 28-36, 2018

seer.perspectivasonline.com.br 
caracteriza notadamente a importância dos Estágios Obrigatórios em Clínicas Escolas, podendo ser caracterizados como um importante mediador para a aquisição dessas competências, na mesma medida que possibilita ser dado um passo importante no processo: aplicar a teoria na prática.

Embora a Terapia Cognitivo-Comportamental pareça relativamente simples, devido ao modelo cognitivo ser direto e objetivo, há muitas tarefas a serem realizadas por sessão. Os terapeutas cognitivos novos precisam ser mais cautelosos, concentrando-se em uma tarefa por vez, seguindo a estrutura da sessão a fim de evitar erros (BECK, 2013). O Estágio Obrigatório na Clínica-Escola segue um padrão devido a burocracia envolvida nos serviços prestados à comunidade. Este padrão é percebido desde o acolhimento do usuário até o processo de avaliação, devolução, intervenção e desligamento. Com isto, os estagiários encontram facilidades ao seguir à risca a estrutura do modelo cognitivo, formando terapeuta qualificados para a atuação no campo profissional.

Por conseguinte, o Estágio Obrigatório na Clínica-Escola possibilitou diversificadas experiências, desde disseminação/aplicação de conhecimentos técnico-científicos em Psicologia, desenvolvimento de processos de ensino-aprendizagem à colaboração entre pares, ratificando assim, a importância de que essas práticas sejam relatadas entre o meio acadêmico.

\section{REFERÊNCIAS}

AMERICAN PSYCHIATRIC ASSOCIATION, A. Manual Diagnóstico e Estatístico de Transtornos Mentais (DSM-5). Porto Alegre: Artmed, 2014.

ANDretTA, I.; OLIVEIRA, M. S. Manual Prático de Terapia Cognitivo-Comportamental. Editora Pearson, 2011.

BECK, J. S. Terapia Cognitivo-Comportamental: Teoria e prática. 2. ed. Porto Alegre: Artmed, 2013.

BRASIL, Ministério da Educação. Diretrizes Curriculares Nacionais para os cursos de graduação em Psicologia, Resolução No 5, de 15 de março de 2011. Brasília, 2011.

BRONFENBRENNER, U. A ecologia do desenvolvimento humano: experimentos naturais e planejados. Porto Alegre: Editora Artes Médicas do Sul, 2002.

CERIONI, R. A. N.; HERZBERG, E. Expectativas de pacientes acerca do Atendimento Psicológico em um Serviço-Escola: da Escuta à Adesão. Psicologia: Ciência e Profissão, Vol. 36, n. 3, p. 597-609, 2016.

CONSELHO FEDERAL DE PSICOLOGIA. Manual de elaboração de documentos produzido pelos psicólogos decorrentes das Avaliações Psicológicas. Brasília: Conselho Federal de Psicologia, Resolução n.7, 14 de junho de 2003. Disponível em <http://site.cfp.org.br/resolucoes/resolucao-n-7-2003/>.

CUNHA, J. A. Manual da versão em português das Escalas Beck. São Paulo: Casa do Psicólogo, 2001.

CRUZ, R. M. Competências Científicas e Profissionais e Exercício Profissional do Psicólogo [Editorial]. Psicologia: Ciência e Profissão, Vol. 36, n. 2, p. 251-254, 2016.

DEL PRETTE, Z. A. Inventário de Habilidades Sociais (IHS-Del Prette): Manual de aplicação, apuração e interpretação. São Paulo: Casa do Psicólogo, 2001.

DE SOUZA, D.A.; MORENO, A. L.; GAUER, G.; MANFRO, G. G.; KOHLLER \& S. H. Revisão sistemática de instrumentos para avaliação de ansiedade na população brasileira. Avaliação Psicológica, Vol. 12, n. 3, p. 397-410, 2013.

DE FARIAS.; FONSECA, F. N.; NERY, L. B. Teoria e Formulação de Casos em Análise Comportamental Clínica. Porto Alegre: Editora Artes Médicas do Sul, 2017.

Persp. online: hum. \& sociais aplicada., Campos dos Goytacazes, 21 (8), 28-36, 2018

seer.perspectivasonline.com.br 
GOMIDE, P. I. Inventário de Estilos Parentais, modelo teórico: manual de aplicação, apuração e interpretação. Petrópolis: Vozes, 2006.

HOFMANN, S. G., SMITS, J. A. J. Cognitive-behavioral therapy for adult anxiety disorders: a meta-analysis of randomized placebo-controlled trials. Journal of Clinical Psychiatry, Vol. 69, n. 4, p. 621-632, 2008.

HOFMANN, S. G., ASNAANI, A., VONK, I. J. J., SAWYER, A. T., FANG, A. The Efficacy of Cognitive Behavioral Therapy: A Review of Meta-analyses. Cognitive Therapy and Research, Vol. 36, n. 5, p. 427440, 2012.

HOFMANN, S. G. Introdução à Terapia Cognitivo-Comportamental Contemporânea. Porto Alegre: Artmed, 2014. $218 \mathrm{p}$.

KNAPP, P., BECK, A. T. Fundamentos, modelos conceituais, aplicações e pesquisa da terapia cognitiva. Revista Brasileira de Psiquiatria, Vol.30, n. 2, p. 54-64, 2008.

MARAVIESKI, S.; SERRALTA, F. B. Características clínicas e sociodemográficas da clientela atendida em uma clínica-escola de Psicologia. Temas em Psicologia, Vol. 19, n. 2, p.481-490, 2011.

DEWES, D., OLIVEIRA, M. S., ANDRETTA, I., MÜHLEN. B. K. V., CAMARGO, J., LEITE, J. C. C. Efetividade da Terapia Cognitivo-Comportamental para os Transtornos do Humor e Ansiedade: uma revisão de revisões sistemáticas. Revista de Psicologia da IMED, Vol. 2, n. 2, p. 385-397, 2010.

SAMPIERI, R. H. ; COLLADO, C. F. ; LUCIO, P. B. Metodologia de la investigación. 6. ed. Álvaro Obregón: Mcgraw-hill, 2014. 588 p.

POWELL, V. B., ABREU, N., OLIVEIRA, I. R., \& SUDAK., D. Terapia Cognitivo-Comportamental da Depressão. Revista Brasileira de Psiquiatria., Vol. 30, n. 3, p. 73-80, 2008.

PRIMI, R. Avaliação Psicológica no Brasil: Fundamentos, Situação Atual e Direções para o Futuro. Psicologia: Teoria e Pesquisa, Brasília, Vol. 26, n. especial , p.25-35, 2010.

ZORTEA, T. C. Notas sobre Superproteção. Vitória: Website Comportamento e Sociedade, 2012. Disponível em https://comportamentoesociedade.com/2012/12/28/notas-sobre-superprotecao.

Persp. online: hum. \& sociais aplicada., Campos dos Goytacazes, 21 (8), 28-36, 2018 seer.perspectivasonline.com.br 